\title{
Nanoparticle Deposition of Fluoropolymer CYTOP via Holographic Femtosecond Laser Processing and Its Biochip Application
}

\author{
Ryo Ozaki ${ }^{1}$, Kotaro Ishida ${ }^{2}$, Eiji Morita ${ }^{2}$ and Yasutaka Hanada ${ }^{1, *}$ \\ 1 Graduate School of Science and Technology, Hirosaki University, 3 Bunkyo-Cho, Hirosaki, \\ Aomori 036-8561, Japan; h19ms608@hirosaki-u.ac.jp \\ 2 Graduate School of Agriculture and Life Science, Hirosaki University, 3 Bunkyo-Cho, Hirosaki, \\ Aomori 036-8561, Japan; ikotaro765@gmail.com (K.I.); moritae@hirosaki-u.ac.jp (E.M.) \\ * Correspondence: y-hanada@hirosaki-u.ac.jp
}

Received: 19 September 2020; Accepted: 13 October 2020; Published: 16 October 2020

Featured Application: The presented holographic femtosecond laser processing technique may achieve a better performance of CYTOP nanoparticle generation compared to the conventional single laser beam processing, and the application of CYTOP nanoparticle deposition lies in the area of biochip and coating industries, where a superhydrophobic surface is required.

\begin{abstract}
The fundamental characteristics of nanoparticle (NP) deposition of the fluoropolymer CYTOP using a femtosecond ( $\mathrm{fs}$ ) laser were investigated. In previous studies, we have demonstrated the microfluidic fabrication of CYTOP, which enables clear microscopic observation of the fluid boundary because of its low refractive index, as well as that of water. In the present work, we generated CYTOP NPs using holographic fs laser processing with a spatial light modulator to demonstrate the capabilities of this functional polymer. We established a deposition technique via five-dot parallel fs laser beam irradiation for fibrous network and monolayer structures composed of CYTOP NPs on the surface of glass slides by manipulating the various fundamental laser processing parameters. The network structure on the glass surface exhibits superhydrophobic behavior, while the monolayer structure performs almost the same wettability as that of CYTOP thin film. After an investigation of the surface features of the NPs deposited onto the glass, the combination of the holographic fs laser deposition and the removal of CYTOP NPs was used to selectively pattern CYTOP NPs on the glass slide for HeLa cell culturing. Consequently, cells were selectively cultured on the glass surface where the laser removal of deposited NPs was carried out.
\end{abstract}

Keywords: nanoparticle; CYTOP; holographic femtosecond laser processing; wettability; selective cell culture; biochip

\section{Introduction}

Within the past 20 years, microelectromechanical system (MEMS) and nanoelectromechanical system (NEMS) technologies have been used to fabricate small biochips for studying various phenomena in the biological sciences [1-4]. The commonly used conventional biochip materials are transparent glasses and polymers, which enable clear observation of reactions in embedded fluidics under a microscope. However, in the case of the fluid boundary, biochips fabricated using conventional materials do not provide sufficiently clear images because of refractive index mismatch between the biochip material and the medium in the fluidic system.

Asahi Glass Co. has developed a commercially available fluoropolymer, CYTOP, with unique properties, including a high degree of transparency toward radiation with wavelengths in the ultraviolet 
to infrared regions and a low refractive index almost equal to that of water. In addition, CYTOP exhibits other properties common to fluoropolymers, such as good heat and chemical resistances and good electrical insulating behavior [5]. Therefore, CYTOP is considered an attractive prospective biochip material. In previous research, we established laser microfabrication techniques for CYTOP and clearly observed aquatic microorganisms near the fluid boundaries in fabricated CYTOP biochips [6-8]. To extend the applications of this polymer, we here attempt to generate CYTOP nanoparticles (NPs) using femtosecond (fs) laser processing and to use the deposited NPs in a biochip application.

NPs are particulate substances that have one dimension less than $100 \mathrm{~nm}$ at least. They have received much attention in a variety of research fields due to their unique properties and potential applications. To date, many methods have been developed for NP synthesis, which are usually based on sol-gel colloidal chemistry, plasma precursor decomposition, electrochemical etching, etc. Among these is the synthesis of NPs by laser ablation, which involves the generation of NPs by laser ablating a solid target. The method is attractive due to the simplicity in equipment configuration and low operation cost compared to the other methods. In particularly, many investigations have been devoted to NP formation through fs laser ablation since the rapid heating and cooling of ablated species caused by the ultrashort pulse duration has the potential to open a new avenue in NP synthesis. For example, Tull et al. [9] have demonstrated the formation of web-like structures composed of Si NP aggregates by the fs laser ablation. Venkatakrishnan et al. [10] fabricated the silica NPs on nanofibre during the silica glass ablation. Nano diamonds with an average particle size of $3.0 \mathrm{~nm}$ were generated by the fs laser ablation of diamond in deionized water [11].

However, one of the major shortcomings of conventional laser processing, including NP synthesis with fs lasers, is low fabrication efficiency. To overcome this problem, holographic laser processing using a spatial light modulator (SLM) has been actively researched to substantially improve the fabrication efficiency by shaping the laser beam into a desired pattern [12-22]. In particular, several papers on holographic fs laser processing have been reported, including papers on surface microfabrication using beam patterns with a desired shape [15-17] and massively parallel beam fabrication [18-20]. Therefore, in the present study, we used holographic fs laser processing to efficiently deposit CYTOP NPs onto glass slides and investigated the fundamental characteristics of the deposited NPs. Following the investigation, we used the holographic laser processing to partially pattern the CYTOP NPs on the glass surface and then used the patterned NPs to selectively culture HeLa cells.

\section{Materials and Methods}

Figure 1 shows a schematic of the holographic fs laser processing setup used to deposit CYTOP NPs onto a glass slide. A fs laser (Ti:sapphire laser: $775 \mathrm{~nm}, 180 \mathrm{fs}$ ) was used as the light source. The repetition rate, which was controlled using a Pockels cell, was varied from $1 \mathrm{~Hz}$ to $1 \mathrm{kHz}$; the laser power was adjusted by neutral density filters, a half-wavelength plate, and a polarizer. The beam was diffracted by a computer-generated hologram displayed on the SLM and was transformed to a desired pattern on the CYTOP substrate (thickness: $200 \mu \mathrm{m}$ ) through a 20× objective lens with a numerical aperture of 0.46 (focal length $=10.0 \mathrm{~mm}$ ). Details of the experimental setup used for holographic fs laser processing are available elsewhere [15]. The laser output before the objective lens and the number of pulses were varied from 3.0 to $8.0 \mu \mathrm{J} /$ pulse and from 1 to 10,000 pulses, respectively. The CYTOP substrate and the glass slide (thickness: $1.3 \mathrm{~mm}$ ) were positioned in parallel, and the movement of each substrate was controlled via a computer-controlled $x y z$ stage. The distance between the CYTOP and the glass slide was also fixed from 100 to $10,000 \mu \mathrm{m}$ during the experiment.

To deposit CYTOP NPs onto the glass slide, five-dot parallel beams with an $8 \mu \mathrm{m}$ pitch were generated by the holographic fs laser processing technique [21] and the surface of the CYTOP substrate was ablated. The morphology of the deposited NPs was observed and analyzed by scanning electron microscopy (SEM) and atomic force microscopy (AFM). After the deposition, the wettability of the glass surface was evaluated by measuring the contact angle of $1 \mu \mathrm{L}$ of pure water dropped onto the glass surface using a pipette. 
After the investigation of the CYTOP NPs, glass slide fabrication via holographic fs laser processing of CYTOP for selective cell culturing was carried out. First, CYTOP NPs were deposited onto a glass slide via five-dot parallel-beam irradiation, as previously described. A line-shaped beam was then also generated by holographic laser processing $[16,22]$ to partially remove the deposited NPs and clearly divide the bare glass surface and the NP-deposited areas.

After fabrication of the glass slides, HeLa cells were cultured on the glass for 1 day at $37^{\circ} \mathrm{C}$ in Dulbecco's modified Eagle's medium (DMEM) containing 100 units $/ \mathrm{mL}$ penicillin, $100 \mu \mathrm{g} / \mathrm{mL}$ of streptomycin, and $10 \%(v / v)$ fetal bovine serum (FBS) in humidified air containing $5 \% \mathrm{CO}_{2}$. After the cell culture, an optical microscope was used in phase-contrast mode to observe the selectively cultured cells on the glass slide.

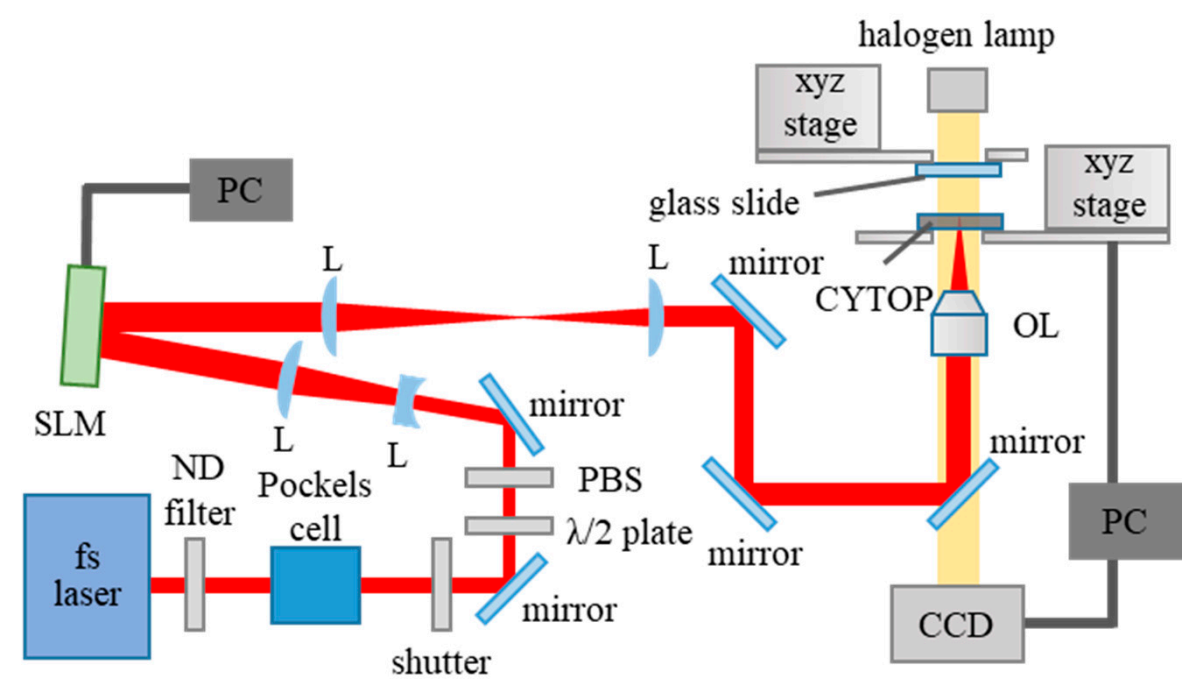

Figure 1. Schematic of the holographic femtosecond (fs) laser processing setup for CYTOP nanoparticle (NP) deposition. (OL: objective lens, L: lens, PBS: polarization beam splitter).

\section{Results}

\subsection{Fundamental Characteristics of CYTOP NP Deposition}

To investigate the fundamental characteristics of the process of CYTOP NP deposition onto glass slides via five-dot parallel fs laser-beam irradiation, the effects of varying experimental conditions such as the CYTOP-glass slide distance and the laser processing parameters were determined. After the deposition, the morphology of the glass surface was characterized by SEM and AFM.

\subsubsection{Influence of the CYTOP-Glass Slide Distance on the NP Deposition}

To elucidate the influence of the CYTOP-glass slide distance on the properties of deposited CYTOP NPs, a glass slide was obliquely placed on the CYTOP substrate, as shown in Figure 2. In this experiment, a fs laser beam with a pulse energy of $5.0 \mathrm{~J} /$ pulse before the objective lens was scanned at the CYTOP surface with the speed of $1500 \mu \mathrm{m} / \mathrm{s}$. After the laser scanning, the NP-deposited areas on the glass surface 100 to $10,000 \mu \mathrm{m}$ from the CYTOP substrate were observed by SEM to characterize the NP deposition tendency at different CYTOP-glass slide distances.

Figure 3 shows SEM images of CYTOP NPs deposited onto the glass slide at different CYTOP-glass distances and the relationship between the diameter of the NPs and the CYTOP-glass slide distance. The average diameter of the NPs was calculated by counting NPs in $200 \times 200 \mathrm{~nm}^{2}$ areas of the glass slide at each distance. The calculation was repeated five times. When the CYTOP-glass slide distance was less than $6000 \mu \mathrm{m}$, NPs approximately $10-100 \mathrm{~nm}$ in diameter agglomerated with other NPs. When the distance exceeded $6000 \mu \mathrm{m}$, particles 10-30 $\mathrm{nm}$ in diameter were uniformly distributed on the glass slide. Figure $3 \mathrm{~b}$ shows that the particle size gradually decreases with increasing CYTOP-glass distance. 
It seems that two different types of surface morphology composed of NP aggregates or relatively small individual NPs can be created on the glass slide depending on the CYTOP-glass slide distance.

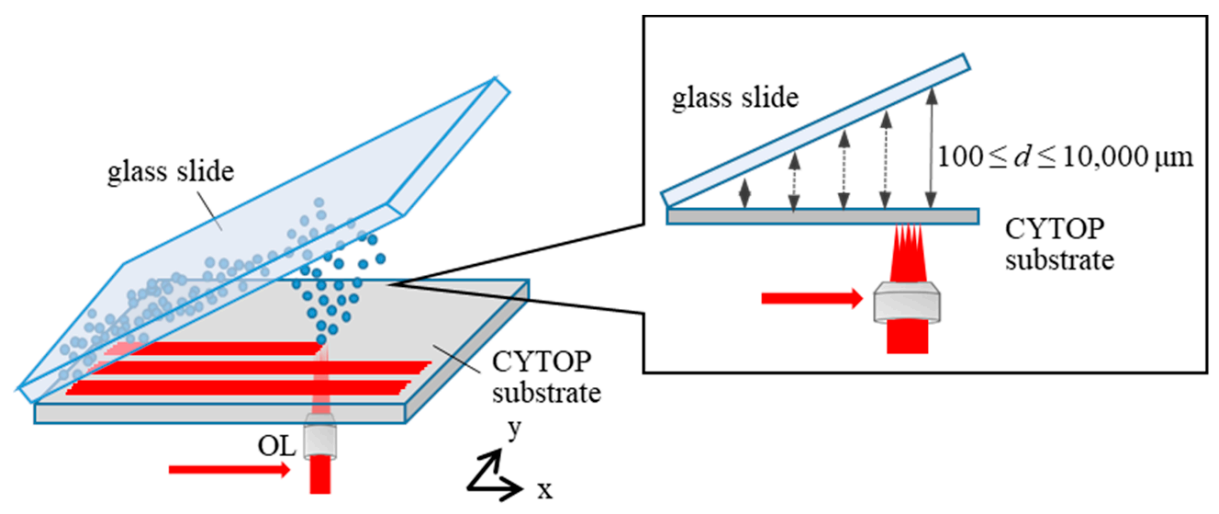

Figure 2. Schematic of the NP deposition process at various CYTOP-glass distances.

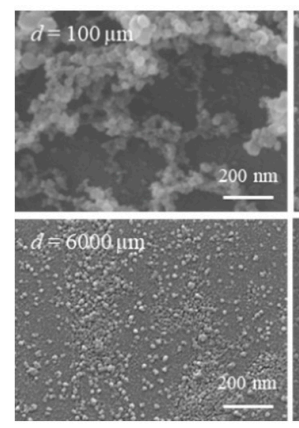

(a)

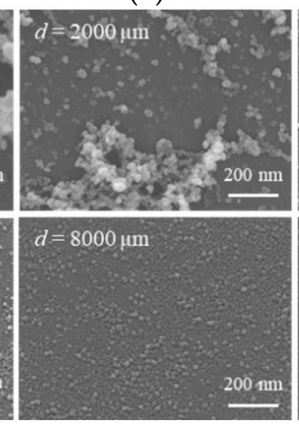

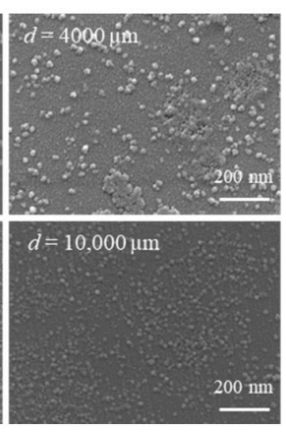

(b)

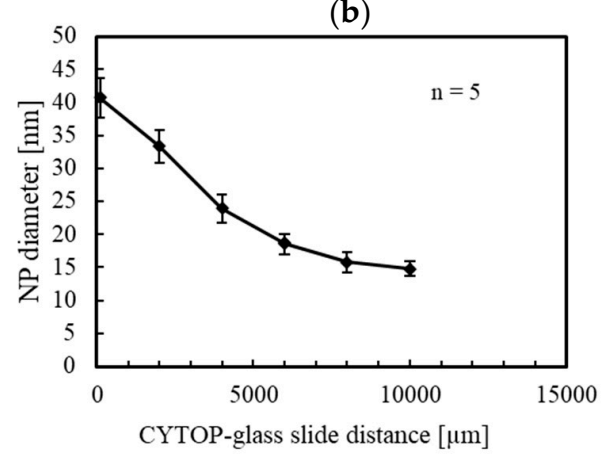

Figure 3. (a) SEM images of deposited NPs with different CYTOP-glass slide distances. (b) NP size as a function of the CYTOP-glass slide distance.

\subsubsection{Influence of Laser Processing Parameters on the NP Deposition}

The morphology of the deposited NPs on the glass also changed depending on laser processing parameters, such as the number of pulses and the pulse energy. Figure 4a shows SEM images of the deposited NPs on the glass when the number of pulses was varied from 1 to 10,000. The lower SEM images show enlarged views of the upper images. In this experiment, the pulse energy and the CYTOP-glass distance were kept constant at $5 \mu \mathrm{J} /$ pulse and $100 \mu \mathrm{m}$, respectively (note that, in this experiment, a glass slide was placed horizontally above the CYTOP substrate, as shown in Figure 1).

As shown in Figure 4a, fibrous network structures composed of NP aggregates were formed on the glass surface, and the pores in the network structures became narrower with an increasing number of pulses. We also conducted the NP deposition at a greater CYTOP-glass slide distance for comparison. Figure $4 \mathrm{~b}$ shows overall (left) and enlarged (upper right) SEM images, a histogram of the NP sizes (middle) obtained from the enlarged SEM, and an AFM image (right) of the glass surface after 10,000 pulse irradiations at a CYTOP-glass distance of $4000 \mu \mathrm{m}$. It can be seen from the images that NPs were distributed fairly uniformly on the glass slide. The average diameter of the NPs was $26.3 \mathrm{~nm}$, with a standard deviation of $\pm 8.6 \mathrm{~nm}$. The NPs were highly monodispersive and close-packed to form a monolayer structure. The surface roughness of the monolayer structure was $18.9 \pm 1.37 \mathrm{~nm}$. 
(a)

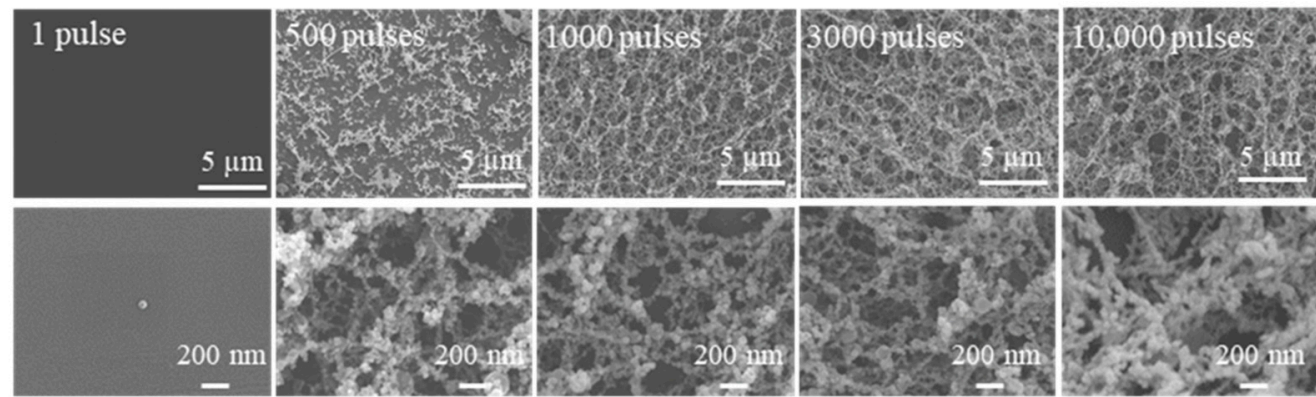

(b)
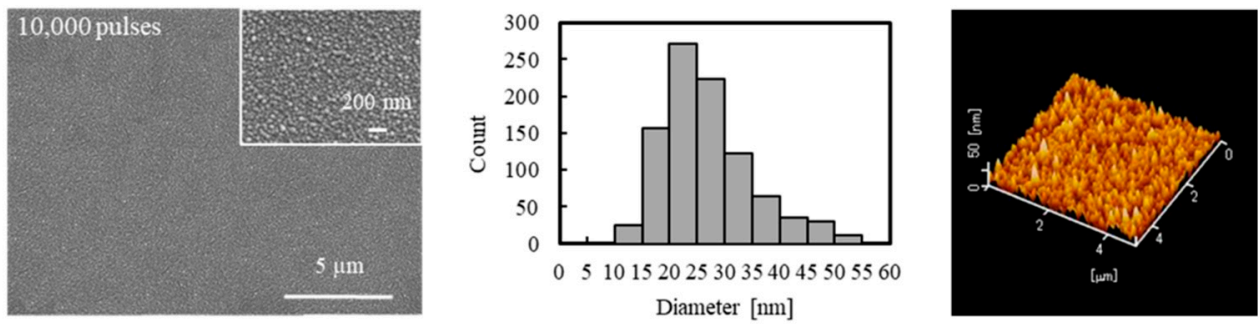

Figure 4. (a) SEM images of NPs deposited with different numbers of pulses. Enlarged views are shown at the bottom. The CYTOP-glass distance was $100 \mu \mathrm{m}$. (b) Low-magnification (left) and enlarged (inset) SEM images, size distribution histogram (middle), and an atomic force microscopy (AFM) image (right) of NPs deposited at a CYTOP-glass distance of $4000 \mu \mathrm{m}$. The number of pulses was 10,000.

Table 1 shows a summary of the glass morphologies after the fs laser irradiation with different CYTOP-glass distances and different numbers of pulses. The pulse energy was maintained at $5 \mu \mathrm{J} / \mathrm{pulse}$. As shown in Table 1, when the CYTOP-glass distance was less than $1000 \mu \mathrm{m}$, network structures tended to deposit on the glass slide. With increasing distance, the monolayer structure was more likely to be formed. Thus, the CYTOP-glass slide distance strongly influenced the morphology of the NP-deposited glass.

Table 1. Effect of the CYTOP-glass distance and the number of pulses on the glass morphology. ( $\square$ : monolayer and $\bigcirc$ : network structure of CYTOP NPs, $\times$ : no CYTOP NP deposition).

\begin{tabular}{cccccc}
\hline No. of Pulses & $\mathbf{1}$ & $\mathbf{5 0 0}$ & $\mathbf{1 0 0 0}$ & $\mathbf{3 0 0 0}$ & $\mathbf{1 0 , 0 0 0}$ \\
Distance $[\boldsymbol{\mu \mathrm { m } ]}$ & & & & & \\
\hline 100 & $\square$ & $\bigcirc$ & $\bigcirc$ & $\bigcirc$ & $\bigcirc$ \\
1000 & $\times$ & $\square$ & $\bigcirc$ & $\bigcirc$ & $\square$ \\
4000 & $\times$ & $\square$ & $\square$ & $\square$ & $\square$ \\
10,000 & $\times$ & $\times$ & $\times$ & $\square$ & $\square$ \\
\hline
\end{tabular}

In addition, we investigated the glass surface after NP deposition at various pulse energies from 3.0 to $8.0 \mu \mathrm{J} /$ pulse, as shown in the SEM images in Figure 5. Enlarged views of the deposited NPs are shown at the bottom. The number of pulses and the CYTOP-glass distance was kept constant at 1000 pulses and $100 \mu \mathrm{m}$, respectively.

The images in Figure 5 show that, when the pulse energy was less than $5 \mu \mathrm{J} /$ pulse, the network structures of NP aggregates gradually formed closed-rings and bridges and became dense with increasing pulse energy. By contrast, when the pulse energy was $8 \mu \mathrm{J} /$ pulse, agglomerates composed of melted NP aggregates were deposited onto the glass slide. 

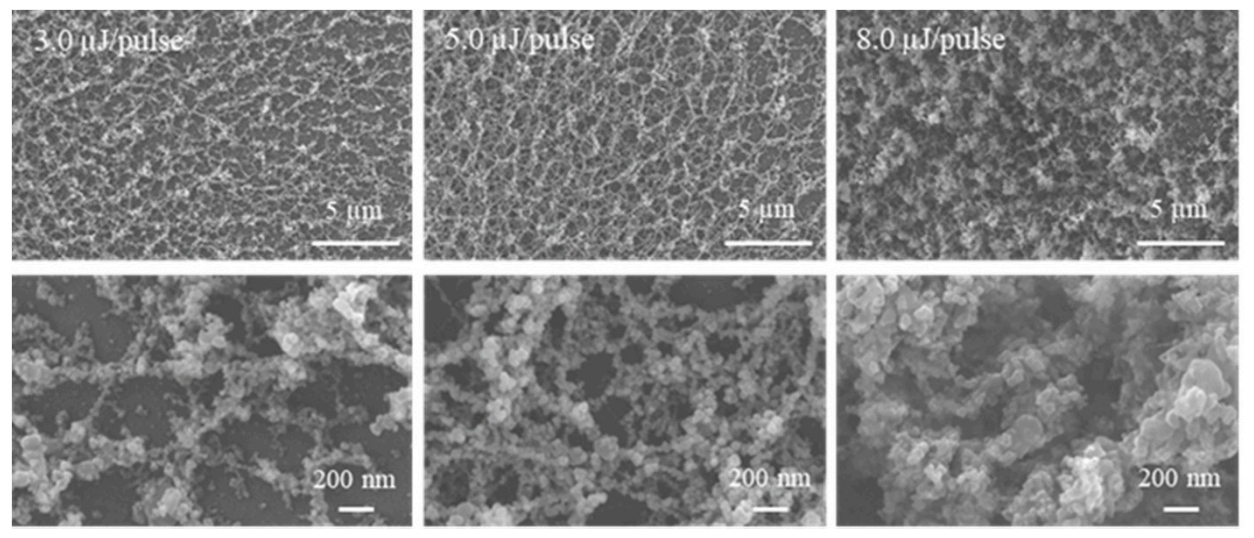

Figure 5. SEM images of NPs on glass slides after fs laser irradiation with different pulse energies.

\subsection{Wettability of the Deposited CYTOP NPs}

The wettability of the network and monolayer structures composed of CYTOP NPs deposited onto glass slides was evaluated. Again, five-dot parallel beams were irradiated onto the same areas of the CYTOP surface to deposit NPs onto the glass slides. The pulse energy and the number of pulses were $5 \mu \mathrm{J} /$ pulse and 10,000, respectively. The CYTOP-glass distance was selected as 100 or $4000 \mu \mathrm{m}$ for deposition of the network or monolayer structure on each glass substrate, respectively. Figure 6 shows an overall SEM image of NPs deposited onto the glass slide following fs laser irradiation at a CYTOP-glass distance of $100 \mu \mathrm{m}$ and a schematic of the NP-distributed areas.
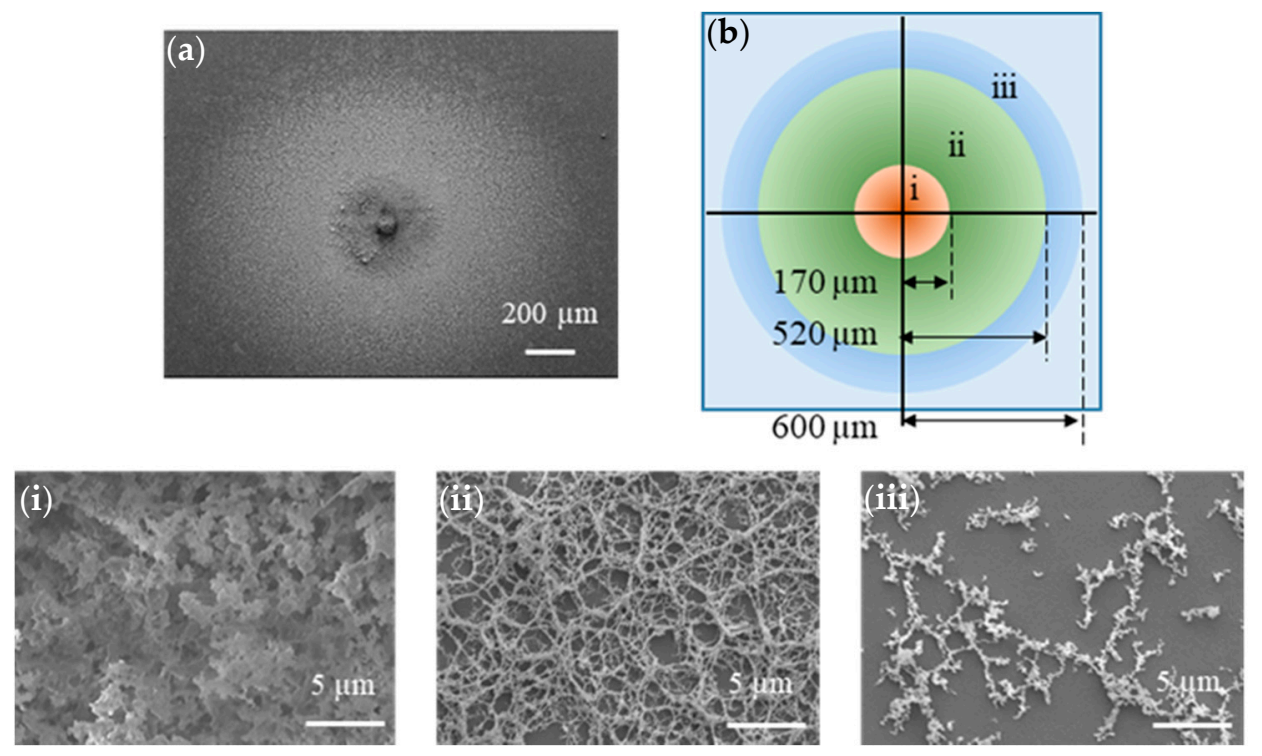

Figure 6. (a) Overall SEM image of deposited NPs on the glass and (b) a schematic of the NP-distributed areas. (i-iii) Enlarged SEM images corresponding to NPs deposited in areas i-iii in subfigure (b).

Figure 6a shows that the CYTOP NPs were deposited radially with a diameter of $1200 \mu \mathrm{m}$ onto the glass slide. Changes in the surface morphology of the deposited NPs are discernible depending on the distance from the center of the NP-distributed areas, as shown in Figure 6b. Figure 6i-iii shows that melted NP aggregates are agglomerated within a distance of $170 \mu \mathrm{m}$ from the center of the NP-deposited areas. Dense network structures are deposited at 170-520 $\mu \mathrm{m}$, and short, chain-like NP aggregates are observed at distances between 520 and $600 \mu \mathrm{m}$; that is, debris of network structures is formed. Thus almost $70 \%$ of the NP-deposited areas are composed of network structures. In the case of a CYTOP-glass distance of $4000 \mu \mathrm{m}$, the monolayer structure (see Figure $4 \mathrm{~b}$ ) is deposited radially, with a diameter of $3000 \mu \mathrm{m}$. 
After NP deposition, we evaluated the wettability of the CYTOP structures by measuring the water contact angle of each structure. Figure 7 shows microscopic images of $1 \mu \mathrm{L}$ water droplets on (a) a normal glass slide, (b) a CYTOP thin film [23], (c) monolayer, and (d) network structures composed of CYTOP NPs deposited onto glass slides. Table 2 summarizes the surface roughness, contact angle, and wettability of each substrate.

(a)

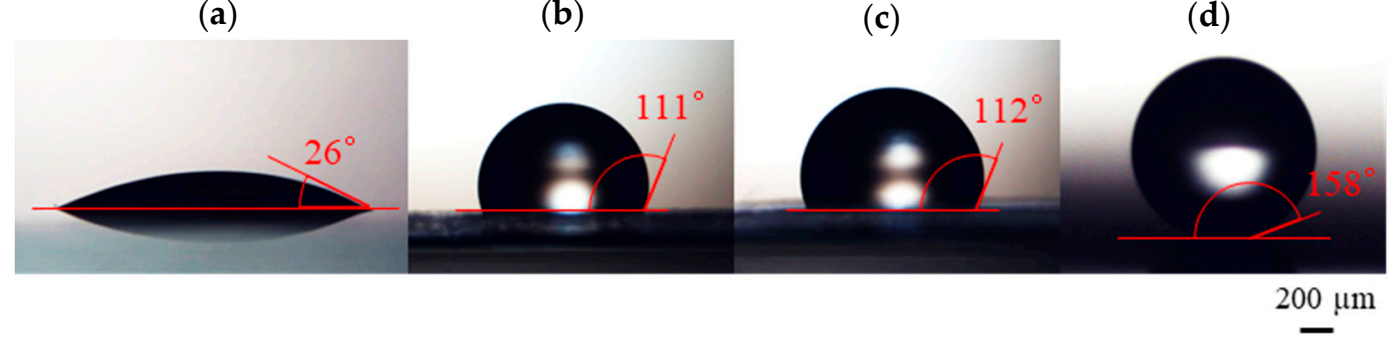

Figure 7. Contact angle measurements of (a) glass slide, (b) CYTOP thin film, (c) monolayer, and (d) network structures of CYTOP NPs deposited onto glass slides.

Table 2. Morphology and wettability measurements of each substrate.

\begin{tabular}{cccc}
\hline Substrate & $\begin{array}{c}\text { Arithmetic Mean Surface } \\
\text { Roughness [nm] }\end{array}$ & Contact Angle [ ${ }^{\circ}$ ] & Wettability \\
\hline (a) Glass slide & $21.2 \pm 0.15$ & 27 & Hydrophilic \\
(b) CYTOP thin film & $20.1 \pm 1.42$ & 112 & Hydrophobic \\
(c) Monolayer structure & $18.9 \pm 1.37$ & 113 & Hydrophobic \\
(d) Network structure & $180.6 \pm 6.10$ & 158 & Superhydrophobic \\
\hline
\end{tabular}

The results in Figure 7 and Table 2 show that a low contact angle was observed for the glass surface, which was expected because the glass slide surface generally exhibits hydrophilic behavior. However, almost the same contact angle was observed on the CYTOP thin film and on the monolayer structure composed of CYTOP NPs on glass, as shown in Figure $7 \mathrm{~b}$ and c. The highest contact angle of $158^{\circ}$ was observed for the network structures deposited onto glass. These results indicate that the wettability of the glass surface can be varied from hydrophilic to superhydrophobic by altering the laser-processing parameters used for CYTOP NP deposition.

\subsection{Selective Cell Culturing on the NP-Deposited Glass}

The results show that deposition of CYTOP NP onto a glass slide can be used to render the glass surface superhydrophobic. We therefore attempted selective cell culturing on a glass slide with CYTOP NPs deposited. Figure 8a shows a schematic of the glass slide fabricated for the selective cell culturing. First, the previously used fs laser irradiation method with five-dot parallel beams was again used to deposit NP network structures onto glass slide. The pulse energy, number of pulses, and the CYTOP-glass distances were $5.0 \mu \mathrm{J} /$ pulse, $100 \mu \mathrm{m}$, and 10,000 pulses, respectively. After the 10,000 pulse irradiations, the glass slide was shifted $350 \mu \mathrm{m}$ along the $x$-direction and the laser irradiation was repeated. As a result, ca. 70\% of the NP-deposited areas were overlapped since the diameter of the deposited areas was measured to be ca. $1200 \mu \mathrm{m}$, as indicated in Figure 6b. This procedure was repeated three times to deposit the network structure over areas of approximately $1.9 \times 1.2 \mathrm{~mm}^{2}$ on the glass slide. After deposition, a line-shaped fs laser beam was also generated via holographic laser processing to partially remove the deposited NPs to divide the glass and NP-deposited areas. The size of the line-shaped fs laser beam was $10 \times 1.5 \mu \mathrm{m}^{2}$ at the focal point. The pulse energy and the scanning speed of the laser beam used to remove the deposited network structures were $3.0 \mu \mathrm{J} / \mathrm{pulse}$ and $100 \mu \mathrm{m} / \mathrm{s}$, respectively. As shown in Figure 8a, the laser beam was scanned $700 \mu \mathrm{m}$ line-by-line along the $y$-direction with a pitch of $5 \mu \mathrm{m}$. This scanning was repeated 10 times to remove $50 \times 700 \mu \mathrm{m}^{2}$ areas of deposited network structures. The laser beam was then shifted $50 \mu \mathrm{m}$ for the next NP removal. 
Figure $8 \mathrm{~b}$ and $\mathrm{c}$ show overall and enlarged SEM images of the fabricated glass slide after the fs laser parallel beam deposition and line-shaped removal of CYTOP NPs.
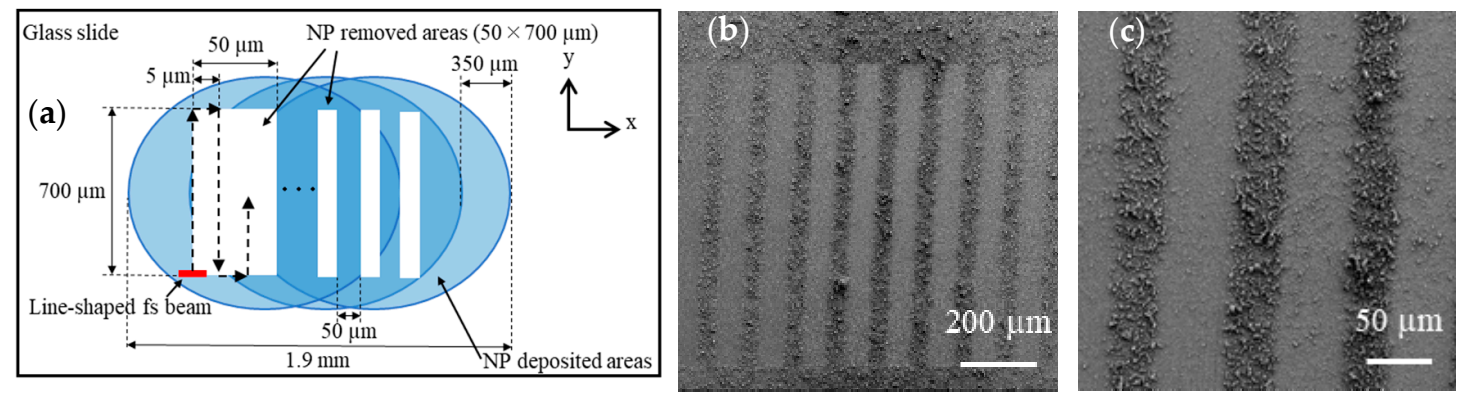

Figure 8. (a) Schematic of the CYTOP NP deposition and removal procedures using holographic fs laser processing on a glass slide. A line-shaped fs laser beam was scanned on the NP-deposited areas to divide the glass and NP-network structures (note that the scales do not relatively agree with each other). SEM images showing (b) an overall view of the fabricated glass slide and (c) an enlarged view of the boundaries between the glass and the deposited network structures.

Figure 8 b shows that deposited network structures on the glass slide were clearly removed by the line-shaped fs laser processing and that clear separation was achieved between the glass surface and the network structures of CYTOP NPs at the boundaries (Figure 8c). Following the glass fabrication process, we attempted to selectively culture HeLa cells on the fabricated glass slide; the results are shown in Figure 9.

(a)

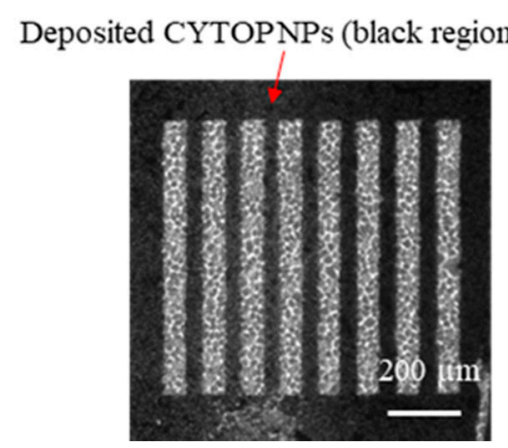

(b)

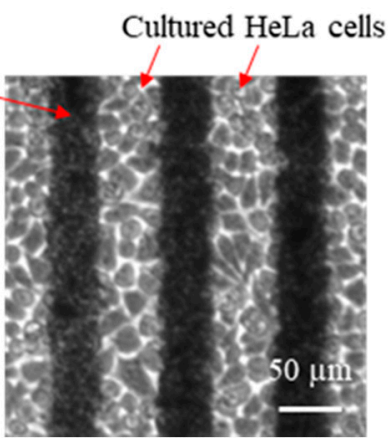

Figure 9. Microscope images showing (a) HeLa cells selectively cultured on the fabricated glass and (b) an enlarged view of the deposited NP-glass boundaries.

Figure 9a shows that HeLa cells were selectively cultured on the glass surface where the line-shaped fs laser removal was carried out. In addition, no cells were cultured on the NP-deposited areas, as shown in the enlarged image of the boundaries between the glass and the deposited CYTOP NPs (Figure 9b). Thus, fs laser deposition of network structures composed of CYTOP NPs imparted functionality to the glass surface and is potentially an attractive tool for cell culture applications.

\section{Discussion}

When the CYTOP-glass distance was short, fibrous network structures composed of NP aggregates were deposited onto the glass slide. At longer CYTOP-glass distances, a monolayer structure of NPs was deposited. In previous studies of fs laser ablation of matter [24,25], when fs laser ablation of CYTOP occurs, critical local expansion dynamics at the focal area lead to the direct fragmentation and ejection of the molten CYTOP. The ejected liquid species from the ablation plume solidifies rapidly and the subsequent growth of the solid fragments will form CYTOP NPs. In fact, agglomerates 
are also formed as a result of collisions of individual NPs during the ablation. Since the network structures composed of NP aggregates were heavier than the individual NPs, the structures could only deposit onto the glass slide when the CYTOP-glass slide distance was short. Only the individual light NPs could be transported longer CYTOP-glass distances, resulting in the deposition of a monolayer structure (Figure 10).

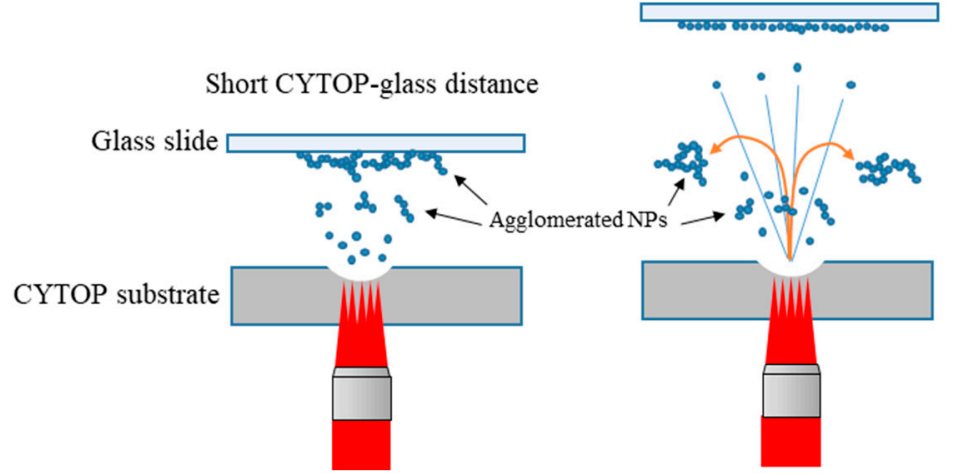

Figure 10. Schematics of the NP deposition via CYTOP ablation, where the deposited structure is dependent on the CYTOP-glass distance.

Furthermore, according to Tan and Venkatakrishnan [26], the pulse frequency plays a critical role in the formation of NP aggregates. They found that network structures of Si NPs were likely to be formed when $\mathrm{MHz}$ fs laser processing was used rather than $\mathrm{kHz}$ processing, even though we observed the same fibrous dense structures of CYTOP NPs in the present work by using $1 \mathrm{kHz}$ fs laser processing. The reason why the $\mathrm{kHz}$ fs laser enables the generation of dense network structures is still unclear; however, we speculate that melted liquid species that eventually form NPs are more likely to be ejected from the ablation plume of CYTOP than from that of Si because the CYTOP is thermoplastic and exhibits a lower melting point than Si. In addition, the holographic fs laser may play an important role in forming network structures. Figure 11 shows SEM images of CYTOP NPs deposited using a single-dot fs laser beam for comparison with the results obtained using five-dot parallel-beam irradiation (Figure 4a). The pulse energy, number of pulses, and the CYTOP-glass distance were $1 \mu \mathrm{J} /$ pulse, 10,000 pulses, and $100 \mu \mathrm{m}$, respectively, and the irradiation was repeated five times by shifting the focal position $8 \mu \mathrm{m}$ along the $x$-direction to be consistent with the irradiation conditions represented in Figure 4a.

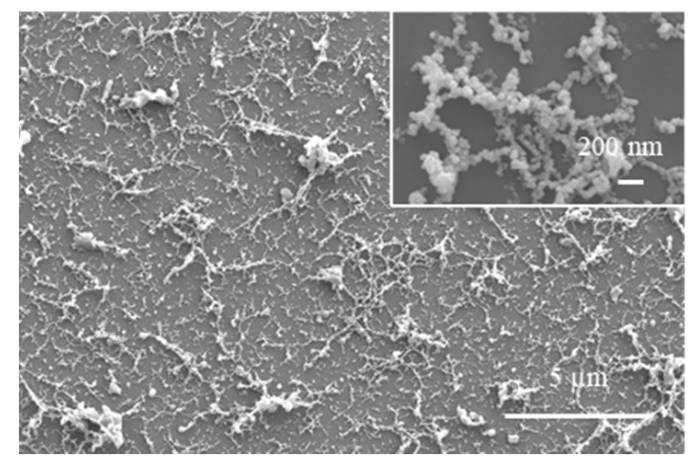

Figure 11. Overall and enlarged (inset) SEM images of CYTOP NPs deposited by single-dot fs laser irradiation.

Figure 11 shows that the short chain-like structures were deposited onto the glass, unlike the dense fibrous network structures observed in Figure 4a. This result suggests that the holographic 
fs laser processing - specifically, the multibeam fs laser irradiation-contributed to the generation of dense fibrous network structures because the collisions and the agglomerates of ejected species from the ablation, which led to the formation of the network structure, were more likely to occur as a consequence of the multiple simultaneous ablations. Given the current experimental results, we cannot conclusively determine the influence of the holographic fs laser processing on NP generation. However, we speculate that these simultaneous particle interactions by holographic laser processing may enable the fabrication of functional nanostructures.

The wettability measurements reveal that the network structures on the glass slide are superhydrophobic, whereas the monolayer structure retains almost the same hydrophobic character as the general CYTOP thin film. The superhydrophobicity of the network structures is supported by the air-trapping Cassie model [27], where air is trapped in the pores of network structures, resulting in a reduction of the actual contact area between the water droplet and the network structures, thus causing superhydrophobic behavior. However, in the case of the monolayer structures with approximately the same small roughness as the general CYTOP thin film (Table 2), water adheres completely to the nanoasperities on the surface, which can be characterized with the Wenzel wetting model [28]. In this case, the monolayer structure was found to exhibit the same wettability as the CYTOP film. These results are consistent with the wettability behavior of various materials for which laser surface micro/nanostructuring was conducted with various laser processing parameters such as wavelength and pulse width [29-31]. For instance, the wettability of the silanized cupper surface with picosecond laser-induced periodic nanostructure gradually varies from hydrophobic to superhydrophobic as the roughness of the periodic structure increases in the order of several hundred $\mathrm{nm}$ [31]. Therefore, physical surface features, namely, the surface roughness/structure, play an important role in the control of wettability.

\section{Conclusions}

We investigated the fundamental characteristics of CYTOP NP deposition by holographic fs laser processing. The fs laser deposition of CYTOP revealed that interweaving fibrous network structures composed of NP aggregates and a monolayer structure of NPs can be formed with appropriate modification of the laser processing parameters. Wettability measurements revealed that the surface of the network structures is superhydrophobic, whereas the monolayer structure exhibits the same hydrophobicity as general CYTOP thin film. The combination of the holographic fs laser deposition method and the removal of CYTOP NPs on the glass slide enabled the selective culturing of HeLa cells on the glass. Thus the CYTOP NP deposition with holographic fs laser processing technique improves the functionality of the surface and can be used, not only for biochip applications, but also in other fields such as coatings.

Author Contributions: Data curation, R.O.; formal analysis, R.O., K.I.; methodology, E.M. and Y.H.; writing — original draft preparation, R.O.; writing—review and editing, R.O., K.I., E.M. and Y.H.; supervision, Y.H.; funding acquisition, Y.H. All authors have read and agreed to the published version of the manuscript.

Funding: This research was funded by JSPS KAKENHI Grant Number 17H02794.

Acknowledgments: We thank Hasegawa and Hayasaki from Utsunomiya University for their fruitful technical assistance with holographic fs laser processing.

Conflicts of Interest: The authors declare no conflict of interest.

\section{References}

1. Ni, M.; Tong, W.H.; Choudhury, D.; Rahim, N.A.A.; Iliescu, C.; Yu, H. Cell Culture on MEMS Platforms. Int. J. Mol. Sci. 2009, 10, 5411-5441.

2. Bhushan, B. Nanotribology and nanomechanics of MEMS/NEMS and BioMEMS/BioNEMS materials and devices. Microelectron. Eng. 2007, 84, 387-412. 
3. Pang, W.; Zhao, H.; Kim, E.S.; Zhang, H.; Yu, H.; Hu, X. Piezoelectric microelectromechanical resonant sensors for chemical and biological detection. Lab. Chip. 2012, 12, 29-44. [CrossRef]

4. Cai, H.; Parks, J.W.; Wall, T.A.; Stott, M.A.; Stambaugh, A.; Alfson, K.; Griffiths, A.; Mathies, R.A.; Carrion, R.; Patterson, J.L.; et al. Optofluidic analysis system for amplification-free, direct detection of Ebola infection. Sci. Rep. 2015, 5, 14494. [CrossRef] [PubMed]

5. CYTOPTM Technical Information. Available online: https://www.agcce.com/cytop-technical-information/ (accessed on 23 July 2020).

6. Hanada, Y.; Ogawa, T.; Koike, K.; Sugioka, K. Making the invisible visible: A microfluidic chip using a low refractive index polymer. Lab. Chip. 2016, 16, 2481-2486. [CrossRef] [PubMed]

7. Ogawa, T.; Hanada, Y. Microfabrication of the UV transparent polymer CYTOP using a conventional pulsed green laser. Appl. Phys. A 2016, 122, 156-161. [CrossRef]

8. Nemoto, K.; Hanada, Y. Etching-Assisted Ablation of the UV-Transparent Fluoropolymer CYTOP Using Various Laser Pulse Widths and Subsequent Microfluidic Applications. Micromachines 2018, 9, 662. [CrossRef] [PubMed]

9. Tull, B.R.; Carey, J.E.; Sheehy, M.A.; Friend, C.; Mazur, E. Formation of silicon nanoparticles and web-like aggregates by femtosecond laser ablation in a background gas. Appl. Phys. A 2006, 83, 341-346. [CrossRef]

10. Venkatakrishnan, K.; Vipparty, D.; Tan, B. Nanofibre fabrication by femtosecond laser ablation of silica glass. Opt. Express 2011, 19, 15770-15776. [CrossRef]

11. Hao, J.; Pan, L.; Gao, S.; Fan, H.; Gao, B. Production of fluorescent nano-diamonds through femtosecond pulsed laser ablation. Opt. Mater. Express 2019, 9, 4734-4741. [CrossRef]

12. Kaakkunen, J.J.J.; Laakso, P.; Kujanpää, V. Adaptive multibeam laser cutting of thin steel sheets with fiber laser using spatial light modulator. J. Laser Appl. 2014, 26, 032008. [CrossRef]

13. Kaakkunen, J.J.J.; Vanttaja, I.; Laakso, P. Fast Micromachining Using Spatial Light Modulator and Galvanometer Scanner with Infrared Pulsed Nanosecond Fiber Laser. J. Laser Micro/Nanoeng. 2014, 9, 37-41. [CrossRef]

14. Kuang, Z.; Li, J.; Edwardson, S.; Perrie, W.; Liu, D.; Dearden, G. Ultrafast laser beam shaping for material processing at imaging plane by geometric masks using a spatial light modulator. Opt. Lasers Eng. 2015, 70, 1-5. [CrossRef]

15. Hayasaki, Y.; Nishitani, M.; Takahashi, H.; Yamamoto, H.; Takita, A.; Suzuki, D.; Hasegawa, S. Experimental investigation of the closest parallel pulses in holographic femtosecond laser processing. Appl. Phys. A 2012, 107, 357-362. [CrossRef]

16. Hasegawa, S.; Shiono, K.; Hayasaki, Y. Femtosecond laser processing with a holographic line-shaped beam. Opt. Express 2015, 23, 23185-23194. [CrossRef] [PubMed]

17. Liu, D.; Wang, Y.; Zhai, Z.; Fang, Z.; Tao, Q.; Perrie, W.; Edwarson, S.P.; Dearden, G. Dynamic laser beam shaping for material processing using hybrid holograms. Opt. Laser Tech. 2018, 102, 68-73. [CrossRef]

18. Hasegawa, S.; Ito, H.; Toyoda, H.; Hayasaki, Y. Massively parallel femtosecond laser processing. Opt. Express 2016, 24, 18513-18524. [CrossRef]

19. Jesacher, A.; Booth, M.J. Parallel direct laser writing in three dimensions with spatially dependent aberration correction. Opt. Express 2019, 18, 21090-21099. [CrossRef]

20. Hasegawa, S.; Hayasaki, Y. Holographic Femtosecond Laser Processing with Multiplexed Phase Fresnel Lenses Displayed on a Liquid Crystal Spatial Light Modulator. Opt. Rev. 2007, 14, 208-213. [CrossRef]

21. Chaen, K.; Takahashi, H.; Hasegawa, S.; Hayasaki, Y. Display method with compensation of the spatial frequency response of a liquid crystal spatial light modulator for holographic femtosecond laser processing. Opt. Commun. 2007, 280, 165-172. [CrossRef]

22. Abe, T.; Hasegawa, S.; Takahashi, H.; Ota, M.; Hayasaki, Y. In-process debris removal in femtosecond laser processing. Appl. Phys. A 2017, 123, 700-706. [CrossRef]

23. Hanada, Y.; Sugioka, K.; Kawano, H.; Tsuchimoto, T.; Miyamoto, I.; Miyawaki, A.; Midorikawa, K. Selective cell culture on UV transparent polymer by $\mathrm{F}_{2}$ laser surface modification. Appl. Surf. Sci. 2009, 255, 9885-9888. [CrossRef]

24. Glover, T.E.; Ackerman, G.D.; Lee, R.W.; Young, D.A. Probing particle synthesis during femtosecond laser ablation: Initial phase transition kinetics. Appl. Phys. B 2004, 78, 995-1000. [CrossRef]

25. Semaltianos, N.G. Nanoparticles by Laser Ablation. Crit. Rev. Solid State Mater. Sci. 2010, 35, $105-124$. [CrossRef] 
26. Tan, B.; Venkatakrishnan, K. Synthesis of fibrous nanoparticle aggregates by femtosecond laser ablation in air. Opt. Express 2009, 17, 1064-1069. [CrossRef] [PubMed]

27. Cassie, A.B.D.; Baxter, S. Wettability of porous surfaces. Trans. Faraday Soc. 1944, 40, 546-551. [CrossRef]

28. Wenzel, R.N. Resistance of solid surfaces to wetting by water. Ind. Eng. Chem. 1936, 28, 988-994. [CrossRef]

29. Riveiro, A.; Soto, R.; Comesaña, R.; Boutinguiza, M.; Vala, J.D.; Quintero, F.; Lusquiños, F.; Pou, J. Laser surface modification of PEEK. Appl. Surf. Sci. 2012, 258, 9437-9442. [CrossRef]

30. Liang, F.; Lehr, J.; Danielczak, L.; Leask, R.; Kietzig, A.M. Robust Non-Wetting PTFE Surfaces by Femtosecond Laser Machining. Int. J. Mol. Sci. 2014, 15, 13681-13696. [CrossRef]

31. Long, J.; Fan, P.; Zhong, M.; Zhang, H.; Xie, Y.; Lin, C. Superhydrophobic and colorful copper surfaces fabricated by picosecond laser induced periodic nanostructures. Appl. Surf. Sci. 2014, 311, 461-467. [CrossRef]

Publisher's Note: MDPI stays neutral with regard to jurisdictional claims in published maps and institutional affiliations.

(C) 2020 by the authors. Licensee MDPI, Basel, Switzerland. This article is an open access article distributed under the terms and conditions of the Creative Commons Attribution (CC BY) license (http://creativecommons.org/licenses/by/4.0/). 